-Communication-

\title{
A New Method for the Preparation of Platinum Electrodes with Single-Crystal (100) Surface
}

\author{
Masae SLMINO and Shigeo SHIBATA*
}

Received Februaly 15, 1988 ; Accepted March 3, 1988

\begin{abstract}
Platinum electrodes with well characterized single-crystal surfaces have easily been obtained from a bead prepared by melting a Pt wire in a oxygen-gas flame ${ }^{11}$. Since then adsorptions and electrocatalysis on Pt electrode have been extensively investigated in atomic level. The single-crystal electrode prepared from the bead, however, is not quite convenient for the electrochemical use, because its working surface is small and required to be used in contact with the surface of electrolyte solution by hanging manner.

The electrode behavior depends on the surface structure rather than on the bulk structure. Therefore, the electrode showing the same behavior as that cut from singlecrystal Pt should also be obtained by covering over a polycrystal Pt substrate with a thin single-crystal surface layer. This method could be applied to electrodes of optional shape and size. It has been found in our laboratory that a thin polycrystal film was preferentially recrystallized to (100) structure by heating for a short time at temperature lower than the melting point of $\mathrm{Pt}$ bulk. The technique described in the present article involves this method.
\end{abstract}

\section{EXPERIMENTAL AND RESULTS}

Smooth polycrystal Pt foiles of $0.05 \mathrm{~mm}$ in thickness and $5 \times 5 \mathrm{~mm}$ in size were used as substrates. Each of these foils was spotwelded with a Pt lead wire. A thin Pt layer was electrodeposited on the substrate from $3 \% \mathrm{H}_{2} \mathrm{PtCl}_{6}$ solution with $20 \mathrm{~mA} \mathrm{~cm}{ }^{-2}$ for $30 \mathrm{~s}$ at $25{ }^{\circ} \mathrm{C}$. Its film showed a bright and slightly black surface and was confirmed to be polycrystal structure by means of the voltammetry and RHEED. The electrodeposited electrode was washed thoroughly with water and then heated at $1200 \sim 1400^{\circ} \mathrm{C}$ in a gas flame for $2 \sim 4$ s. The heated electrode was quickly dipped in water to prevent the formation of oxide on the surface. This consecutive process was repeated on the same electrode. Fig. 1 shows voltammetry curves for the substrate and for new surfaces formed by repeating the electroplating and heating. Every time the new thin film was piled on the surface, peak I of the voltammetry curve decreased and peak II grew rapidly. These changes of the curve suggests a preferential (100) orientation in the newly piled surface layer. The repetition of the electroplating and heating promotes this orientation. The well defined (100)-

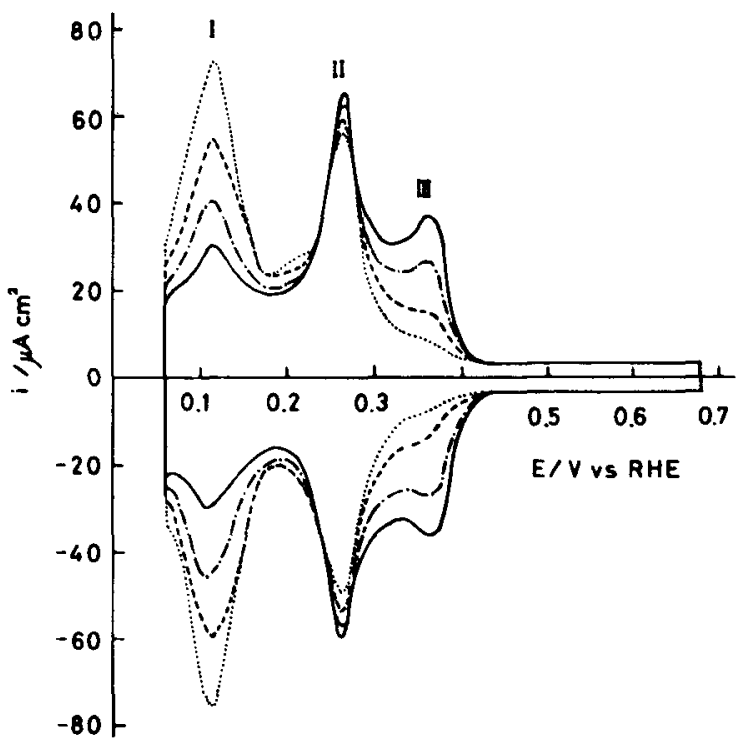

Fig. 1 Voltammetry curve at $50 \mathrm{mV} \mathrm{s} \mathrm{s}^{-1}$ $0.5 \mathrm{M} \mathrm{H}_{2} \mathrm{SO}_{4}$ at $25{ }^{\circ} \mathrm{C}$.

$(\cdots . .$.$) ; for the substrate.$

$(---)$; after electroplating and heating.

(--) and (--) ; after repeating.

3 and 5 times, respectively, the same treatment as above. 


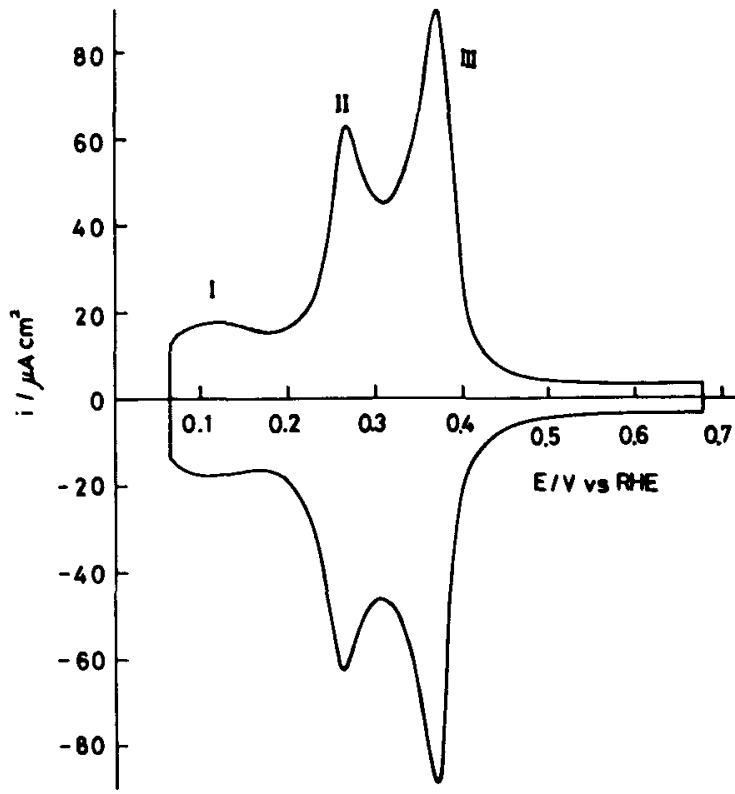

Fig. 2 Voltammetry curve for the well defined (100) surface obtained by the repetition of the electro-plating and heating 20 times.

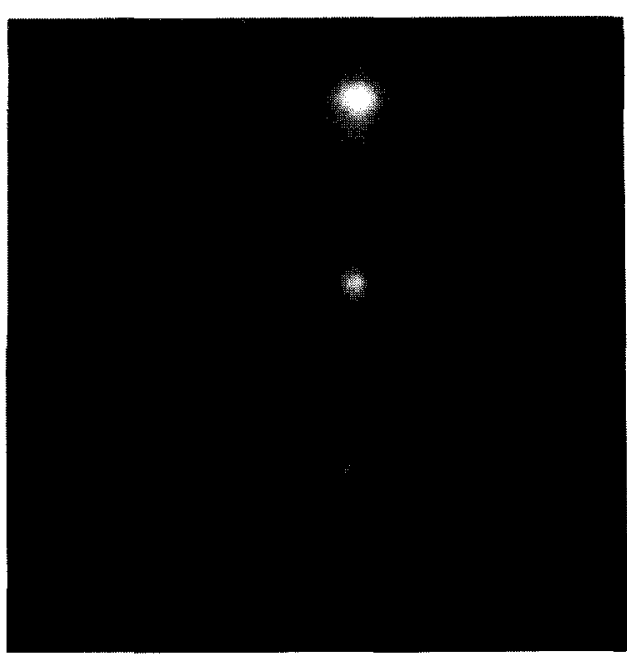

Fig. 3 RHEED pattern from the Pt surface prepared by the repetition of electroplating and heating 20 times. Energy of electrons; $50 \mathrm{keV}$.

surface was completed by repeating this consecutive process more than 20 times. As can be seen from the voltammetry curve for this electrode (Fig.2), peak I disappeared and peak III grew to a new main peak. The shape of this voltammetry curve and potential values of peaks II and III are in good agreement with respective those for (100) single crystal electrode reported by Clavilier ${ }^{2)}{ }^{3)}$. The surface structure was confirmed also by RHEED as shown in Fig. 3. Electron diffraction patterns expected from (100) structure were observed all over the surface.

The current efficiency of the electrodeposition of $\mathrm{Pt}$ from $\mathrm{H}_{2} \mathrm{PtCl}_{6}$ solution under the above described condition was about $50 \%$. Therefore. the total thickness of the new surface layer formed by repeating the electroplating and heating 20 times was estimated to be $1 \mu \mathrm{m}$. Its surface was very smooth, and the roughness factor was on $1 \mathrm{y} 15 \%$ more than that of the substrate.

When the prepared (100) electrode was subjected to the repeating oxidation and reduction by a cyclic potential sweep or by a galvanostatic polarization with alternating current in oxygen potential region, its surface changed gradually to porycrystalline structure. This electrode, however, was recovered to the original single-crystal surface by heating in the gas flame. For example, the voltammetry curve for the electrode which was subjected to the cyclic potential sweep between $0.05 \mathrm{~V}$ to $1.5 \mathrm{~V}$ vs. RHE at $3.5 \mathrm{~Hz}$ for $170 \mathrm{~s}$ in $0.5 \mathrm{H}_{2} \mathrm{SO}_{4}$ showed $70 \%$ decrease in height of peak III and the remarkable growth of peak I. This electrode, after heating at $1200 \sim 1400{ }^{\circ} \mathrm{C}$ for $2 \sim 4 \mathrm{~s}$, showed the same voltammetry curve as that of the (100) surface. This recrystallization was also confirmed by RHEED.

When the electrode was prepared under conditions different from the above described ones, it was difficult to obtain the well-defined (100) surface. Especially, at heating temperature lower than $1000^{\circ} \mathrm{C}$ the electrodeposited Pt layer, even if it was prepared under any conditions, hardly recrystallized to the (100) structure.

\section{REFERENCES}

1) J.Clavilier, R. Faure, G. Guinet and R. Durand, J. Electroanal. Chem., 107, 205 (1980) .

2) J.Clavilier and D.Armand, J. Electroanan, Chem., 199, $187(1986)$.

3) D. Armand and J.Clavilier, J. Electroanal. Chem. , $\overline{225}, 205(1987)$. 